\title{
MACRÓFITAS AQUÁTICAS COMO BIOINDICADORAS DA QUALIDADE DA ÁGUA DOS ARROIOS DA RPPN MARAGATO
}

\author{
Carla Grasiele Zanin Hegel* \\ Evanisa Fátima Reginato Quevedo Melo**
}

RESUMO: A poluição dos ambientes aquáticos tem provocado efeitos sobre as comunidades de seres vivos, os quais podem ser utilizados para avaliar as modificações da qualidade do meio. As macrófitas aquáticas desempenham diversas funções nos ecossistemas, entre elas a capacidade de absorverem o excesso de nutrientes e poluentes da água. Este trabalho teve como objetivo verificar a presença, a riqueza e a frequência de macrófitas aquáticas de um trecho do Arroio do Valinho e do Arroio Pinheiro Torto, e avaliá-las como possíveis bioindicadoras da qualidade das águas da Reserva Particular do Patrimônio Natural Maragato (RS). Não foi encontrada nenhuma espécie de macrófita aquática para o trecho do Arroio do Valinho, enquanto no trecho do Arroio do Pinheiro Torto foram descritas oito espécies. A frequência foi avaliada em um transecto de $200 \mathrm{~m}$ no ponto de encontro dos arroios, onde a espécie Tripogandra diuretica mostrou $100 \%$ de frequência, estendendo-se pela encosta ao longo de toda a margem do trecho analisado. A análise de substrato do fundo apresentou teores de $\mathrm{P}$ muito alto e $\mathrm{K}$, alto; bem como $\mathrm{Mn}, \mathrm{Zn}$ e $\mathrm{Cu}$ acima dos valores de referência. A análise de macronutrientes e micronutrientes, em tecido vegetal na espécie Egeria densa (mais frequente no leito), revelou altos valores de $\mathrm{N}, \mathrm{Fe}, \mathrm{Mn}$ e Zn. Este estudo é uma análise complementar à dos parâmetros físicos, químicos e biológicos de qualidade de água.

PALAVRAS-CHAVE: Ambiente impactado; Biodiversidade; Plantas aquáticas; Recursos hídricos.

\section{WATER MACROPHYTES AS BIO-INDICATORS OF WATER QUALITY IN THE RPPN MARAGATO STREAMS}

ABSTRACT: The pollution of water sources affects communities of living beings which may be used to assess modifications in the quality of the environment.

\footnotetext{
Mestrado em andamento em Ecologia pela Universidade Regional Integrada do Alto Uruguai e das Missões (URI) Campus Erechim, Brasil; E-mail: carlinhahehe@yahoo.com.br

** Doutora em Agronomia pela Universidade Federal de Santa Maria (UFSM); Docente titular da Universidade de Passo Fundo (UPF), Brasil
} 
Water macrophytes have several functions in the ecosystems, among which may be mentioned the fact that they absorb excess of nutrients and water pollutants. Current research assesses the presence, richness and frequency of water macrophytes in a stretch of the Arroio do Valinho and Arroio Pinheiro Torto and evaluates them as bio-indicators of water quality in the Reserva Particular do Patrimônio Natural Maragato in the state of Rio Grande do Sul, Brazil. Whereas no macrophyte species occurred for the stretch of Arroio do Valinho, eight species were described for the stretch of Arroio do Pinheiro Torto. Frequency was evaluated within a 200 meter transect at the streams' meeting site where the species Tripogandra diuretica had $100 \%$ frequency, from the banks throughout the entire margin of the stretch under analysis. The bottom substrate had very high $\mathrm{P}$ and $\mathrm{K}$ rates, with $\mathrm{Mn}, \mathrm{Zn}$ and $\mathrm{Cu}$ exceeding reference rates. Analyses of macro- and micro-nutrients in the vegetal tissue of Egeria densa (more frequent in the river bed) revealed high N, Fe, Mn and $\mathrm{Zn}$ rates. Current analysis complements investigation on the physical, chemical and biological parameters of water quality.

KEY WORDS: Impacted environment; Biodiversity; Water plants; Water resources.

\section{INTRODUÇÃO}

A degradação da qualidade da água ao longo do século XX tornou-se uma das principais preocupações sociais (MAGALHÃES, 2007). A poluição dos ambientes aquáticos, como consequência das atividades antrópicas, tem provocado perturbações nas comunidades de seres vivos em termos de abundância, de riqueza, de comportamento e de reações fisiológicas (AGENCES DE L'EAU, 1993), pois as condições do meio aquático produzem efeitos sobre as espécies que vivem nestes habitats (LÉVÊQUE, 1996).

Atualmente a avaliação da qualidade da água pode ser feita por meio de processos físicos, químicos e biológicos (FONTOURA, 1984; CORTES et al., 2002). Através da análise biológica é possível avaliar os efeitos da poluição sobre as comunidades de seres vivos (CORTES et al., 2002), já que a sua presença, abundância e/ou riqueza permitem inferir ou detectar modificações da qualidade do meio (AGENCES DE L'EAU, 1993), que se manifesta após o limite da capacidade de adaptação das espécies (LÉVÊQUE, 1996). As tendências internacionais no 
campo de gestão da água mostram uma crescente utilização de bioindicadores e os recomendam como complemento às informações sobre os demais parâmetros analisados quanto à qualidade das águas (MAGALHÃES, 2007).

Os indicadores biológicos ou bioindicadores são organismos, populações ou comunidades cuja presença, quantidade e distribuição podem indicar a magnitude de alguns impactos ambientais em um ecossistema aquático (KOVÁCS, 1992; CALLISTO; GONÇALVES, 2002; CALLISTO et al., 2005), visto que devido à sua sensibilidade podem ser tolerantes ou intolerantes às alterações do meio. Destacamse como organismos bioindicadores de qualidade da água algumas espécies de bactérias, de protozoários, de macroalgas e microalgas, de macrófitas aquáticas, macroinvertebrados, microinvertebrados e peixes.

As macrófitas (macro = grande; fita $=$ planta) são formas macroscópicas de vegetação aquática (WETZEL, 1993), visíveis a olho nu, cujas partes fotossintetizantes ativas estão permanentemente, ou por diversos meses, todos os anos, total ou parcialmente submersas em água doce ou salobra, ou ainda flutuantes na mesma (IRGANG; GASTAL JR, 1996). Podem ser utilizadas como indicadores da qualidade da água, uma vez que desempenham importantes funções nos ecossistemas aquáticos participando da ciclagem e estocagem de nutrientes, da formação de detritos orgânicos, do controle da poluição e da eutrofização artificial das águas (ESTEVES; CAMARGO, 1986; POTT; POTT, 2000). Além disso representam uma fonte de matéria orgânica para bactérias, invertebrados e vertebrados, tanto enquanto vivas como mortas (detritos) e, principalmente, alteram a estrutura espacial dos habitats (ESTEVES, 2011). Em rios e riachos influenciam na sedimentação e na retenção de nutrientes, nas características físicas e químicas da água e, em alguns casos, podem afetar a velocidade do fluxo da água (ESTEVES, 1998). Scremin-Dias et al. (1999) definem como macrófitas aquáticas as macroalgas, musgos, pteridófitas e angiospermas, originárias do ambiente terrestre com adaptações para a vida na água; ou seja, aquelas que ocorrem tanto em ambientes mais secos da borda dos ambientes aquáticos como dentro da água.

Os principais métodos envolvidos nas análises com esses bioindicadores abrangem o levantamento de espécies, a avaliação das modificações na riqueza e os índices de diversidade; abundância de organismos resistentes; perda de 
espécies sensíveis; medidas de produtividade primária e secundária; sensibilidade a concentrações de substâncias tóxicas (ensaios ecotoxicológicos), entre outros (BARBOUR et al., 1999).

Conforme Cervi et al. (2009), os estudos sobre as macrófitas aquáticas brasileiras são escassos e estão concentrados em poucas áreas do país (CORDAZZO; SEELINGER, 1988; NOTARE, 1992; IRGANG; GASTAL, 1996; SCREMIN-DIAS et al., 1999; POTT; POTT, 2000; POMPÊO; MOSCHINI-CARLOS, 2003; THOMAZ; BINI, 2003).

Segundo Diniz et al. (2005), no Brasil, alguns estudos têm sido realizados para examinar o papel das macrófitas na melhoria da qualidade da água. Os primeiros estudos foram desenvolvidos por Manfrinato (1989), que verificou a eficiência da Eichbornia crassipes na descontaminação das águas do Rio Piracicaba (SP). Figueroa (1996) avaliou as funções ecológicas das macrófitas na represa do Lobo (SP). Luciano (1996) analisou sua importância nos processos de retenção e liberação de nutrientes na represa Jurumirim (SP). Moraes (1999) estimou o estoque de elementos químicos na vegetação do reservatório de Salto Grande (SP). Lopes-Ferreira (2000) avaliou sua eficiência na redução de coliformes e de nutrientes durante um ciclo hidrológico. Porém, ainda são poucos os estudos realizados com as macrófitas aquáticas como bioindicadoras da qualidade da água, especialmente no Rio Grande do Sul. Estudos em ambientes lóticos são ainda mais escassos. Thomaz e Bini (2003) colocam que essa escassez pode ser explicada pelo fato de que os ecossistemas lóticos brasileiros, em geral, são pouco colonizados por macrófitas aquáticas em virtude da variação acentuada dos níveis de água, dos elevados valores de turbidez e correnteza, dentre outros fatores. Algumas exceções são encontradas nas regiões próximas à Serra da Bodoquena (SCREMIN-DIAS et al., 1999) e em rios do litoral sul paulista (CAMARGO; FLORENTINO, 2000).

Este trabalho teve como objetivo verificar a presença, a riqueza e a frequência de macrófitas aquáticas em um trecho do Arroio do Valinho e do Arroio Pinheiro Torto, e avaliá-las como possíveis bioindicadoras da qualidade da água da Reserva Particular do Patrimônio Natural Maragato, município de Passo Fundo (RS). 


\section{METODOLOGIA}

\section{1 ÁREA DE ESTUDO}

O estudo foi realizado nos trechos dos arroios do Valinho e do Pinheiro Torto, situados na Reserva Particular do Patrimônio Natural (RPPN) Maragato (28¹4'28,92S; $\left.52^{\circ} 27 ’ 26,38 \mathrm{~W}\right)$, localizada no município de Passo Fundo, Rio Grande do Sul, Brasil. A propriedade possui uma área total de 182 hectares dos quais 41,56 hectares são destinados à preservação de uma rica diversidade de fauna e flora remanescentes da Floresta Ombrófila Mista do Estado, além de proteger áreas úmidas, nascentes e córregos que formam a Bacia Hidrográfica do Alto Jacuí. Encontra-se na formação da Serra Geral e o solo da região é classificado como Latossolo vermelho distrófico típico em transição com Nitossolo vermelho distroférrico (BRASIL, 2011). A RPPN encontra-se circundada por áreas com atividade agrícola e industrial, devido à sua proximidade com o distrito industrial do município de Passo Fundo (Figura 1). Foi oficialmente reconhecida como unidade de conservação em 15 de outubro de 2007 sob a Portaria ICMBio n ${ }^{\circ}$ 14/2007.
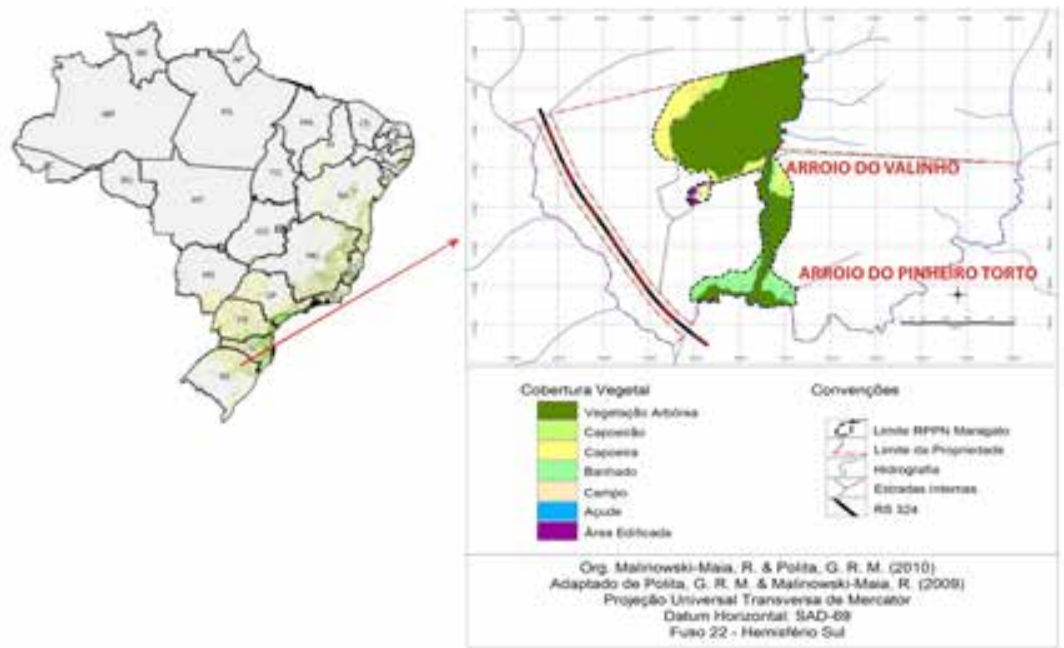

Figura 1. Mapa localizando o município de Passo Fundo no Brasil. Mapa de cobertura vegetal da RPPN Maragato e Arroios do Valinho (ao lado) e Arroio do Pinheiro Torto (abaixo) da figura Fonte: Plano de Manejo da RPPN Maragato (2011) adaptado por Hegel (2014). 
O Arroio Valinho tem suas nascentes localizadas em meio às áreas com desenvolvimento de atividades agrícolas e áreas protegidas, uma delas pertencente à Prefeitura Municipal de Passo Fundo e outra da RPPN Maragato. O trecho no qual foi desenvolvido o presente estudo possui largura entre $80 \mathrm{~cm}$ e $1,50 \mathrm{~m}$ e profundidade de 10 a $60 \mathrm{~cm}$, com ambas as margens preservadas e protegidas pelo remanescente de floresta da RPPN.

O Arroio do Pinheiro Torto nasce em meio a uma área urbanizada, situada próxima ao distrito industrial. Ao longo de seu percurso recebe parte do esgoto doméstico e industrial do município até o limite com a área da RPPN Maragato, seguindo pela área industrial. $O$ trecho estudado possui largura entre 1,20 e $5 \mathrm{~m} \mathrm{e}$ profundidade de $40 \mathrm{~cm}$ a $2 \mathrm{~m}$, com apenas uma das margens preservada.

As águas do Arroio do Valinho se encontram com as águas do Arroio do Pinheiro Torto $\left(28^{\circ} 24^{\prime} 73,91 S ; 52^{\circ} 45^{\prime} 89,89 \mathrm{~W}\right)$ na divisa da RPPN Maragato com a área da Associação do Sindicato dos Trabalhadores das Indústrias da Construção e do Mobiliário de Passo Fundo.

\subsection{MATERIAIS E MÉTODOS}

A amostragem das espécies de macrófitas ocorreu na estação quente do ano, nos dias 30 de dezembro de 2013 na área do Arroio do Valinho, e 21 de janeiro de 2014 na área do Arroio do Pinheiro Torto. Foram utilizadas diferentes metodologias para avaliar a presença, riqueza e frequência das espécies, além de análises de tecidos vegetais e do substrato do leito.

1) Riqueza de espécies: Foram estabelecidos dois transectos: um no Arroio do Valinho, medindo aproximadamente $1400 \mathrm{~m}$ de comprimento, localizado dentro da área da RPPN Maragato, e um no Arroio do Pinheiro Torto, com aproximadamente $700 \mathrm{~m}$ de comprimento na divisa com a área da RPPN Maragato. Por meio de caminhadas, ao longo dos trechos selecionados de cada arroio, verificou-se a presença de espécies de macrófitas aquáticas, as quais quando encontradas foram coletadas com auxílio de uma pá e acondicionadas em sacos plásticos para posterior identificação. As amostras foram encaminhadas ao Herbário da Universidade de Passo Fundo (RSPF) 
para serem desidratadas em estufa, conservadas em forma de exsicatas, tombadas no acervo e identificadas com base na literatura especializada.

Para a classificação das formas biológicas das macrófitas aquáticas utilizouse as categorias propostas por Pedralli (1990), com o reconhecimento de sete tipos: submersa fixa - enraizadas e crescem totalmente submersas na água; submersa livre - permanecem flutuando submersas na água; flutuante fixa - são enraizadas e com folhas flutuando na superfície da água; flutuante livre - permanecem flutuando com as raízes abaixo da superfície da água; anfíbia - plantas geralmente de margens; emergente - enraizadas com folhas emergindo parcialmente; epífita - se desenvolvem sobre outra planta.

2) Frequência: A frequência de espécies encontradas foi estimada em um transecto de $200 \mathrm{~m}$ de comprimento ( $50 \mathrm{~m}$ à montante e $150 \mathrm{~m}$ à jusante), no Arroio do Pinheiro Torto, contemplando o ponto de encontro dos dois arroios.

3) Análise de tecido vegetal da espécie mais frequente do leito: Uma amostra da espécie mais frequente encontrada na coluna de água foi coletada com auxílio de pá e encaminhada ao Laboratório de Solos da Universidade de Passo Fundo, Passo Fundo (RS), para a análise de tecido vegetal quanto à caracterização química de macronutrientes e micronutrientes. Tal procedimento se deu pelo método de Tedesco et al. (1995) e valores de referência conforme estabelecido pela Comissão de Química e Fertilidade do Solo - RS/SC (2004).

4) Análise do substrato do leito: Para a análise de substrato do leito, foi coletada uma amostra junto à espécie mais frequente encontrada na coluna de água. O material também foi encaminhado ao Laboratório de Solos da Universidade de Passo Fundo, para caracterização química de macronutrientes e micronutrientes, segundo o método de Tedesco et al. (1995) e valores de referência conforme estabelecido pela Comissão de Química e Fertilidade do Solo - RS/SC (2004).

\section{RESULTADOS}

Para o trecho analisado do Arroio do Valinho não foi encontrada nenhuma 
espécie de macrófita aquática. Já no trecho analisado do Arroio do Pinheiro Torto, foram descritas oito espécies de oito famílias (Tabela 1, Figura 2).

Tabela 1. Macrófitas aquáticas no Arroio do Pinheiro Torto na área da RPPN Maragato. $\mathrm{N}=$ número de vezes na amostragem. $\%=$ porcentagem de amostras presentes

\begin{tabular}{|c|c|c|c|c|c|}
\hline Família/Espécie & Nome popular & $\begin{array}{c}\text { Situação } \\
\text { no RS }\end{array}$ & $\begin{array}{l}\text { Modo de } \\
\text { vida }\end{array}$ & $\mathbf{N}$ & $\%$ \\
\hline Araceae Lemna minor L. & Lentilha d'água & nativa & $\begin{array}{l}\text { Flutuante } \\
\text { livre }\end{array}$ & 1 & 0,5 \\
\hline $\begin{array}{l}\text { Commelinaceae Tripogandra } \\
\text { diuretica (Mart.) Handlos }\end{array}$ & $\begin{array}{l}\text { Trapoeraba, } \\
\text { Erva-se-santa- } \\
\text { luzia } \\
\end{array}$ & nativa & anfíbia & - & 100 \\
\hline $\begin{array}{l}\text { Convolvulaceae Ipomoea cairica } \\
\text { (L.) Sweet }\end{array}$ & $\begin{array}{l}\text { Ipoméia, } \\
\text { Campainha, } \\
\text { Corda-de-viola }\end{array}$ & nativa & anfíbia & 1 & 0,5 \\
\hline $\begin{array}{l}\text { Hydrocharitaceae Egeria densa } \\
\text { Planch. }\end{array}$ & $\begin{array}{c}\text { Anacharis, } \\
\text { Elódea }\end{array}$ & nativa & $\begin{array}{l}\text { submersa } \\
\text { fixa }\end{array}$ & 85 & 42,5 \\
\hline Lamiaceae Não identificada & - & - & emergente & 1 & 0,5 \\
\hline $\begin{array}{l}\text { Linderniaceae Micranthemum } \\
\text { micrantbemoides (Walter ex J.F. } \\
\text { Gmel.) Blake }\end{array}$ & - & nativa & $\begin{array}{l}\text { submersa } \\
\text { livre }\end{array}$ & 42 & 21 \\
\hline $\begin{array}{l}\text { Poaceae Brachiaria plantaginea } \\
\text { (Link) Hitch. }\end{array}$ & Capim-papuã & nativa & anfíbia & 4 & 2 \\
\hline $\begin{array}{l}\text { Pontederiaceae Heteranthera } \\
\text { reniformis Ruiz et Pav. }\end{array}$ & $\begin{array}{l}\text { Agrião-do-brejo, } \\
\text { Aguapé }\end{array}$ & nativa & $\begin{array}{l}\text { flutuante } \\
\text { livre }\end{array}$ & 8 & 4 \\
\hline
\end{tabular}

Fonte: Dados da pesquisa 


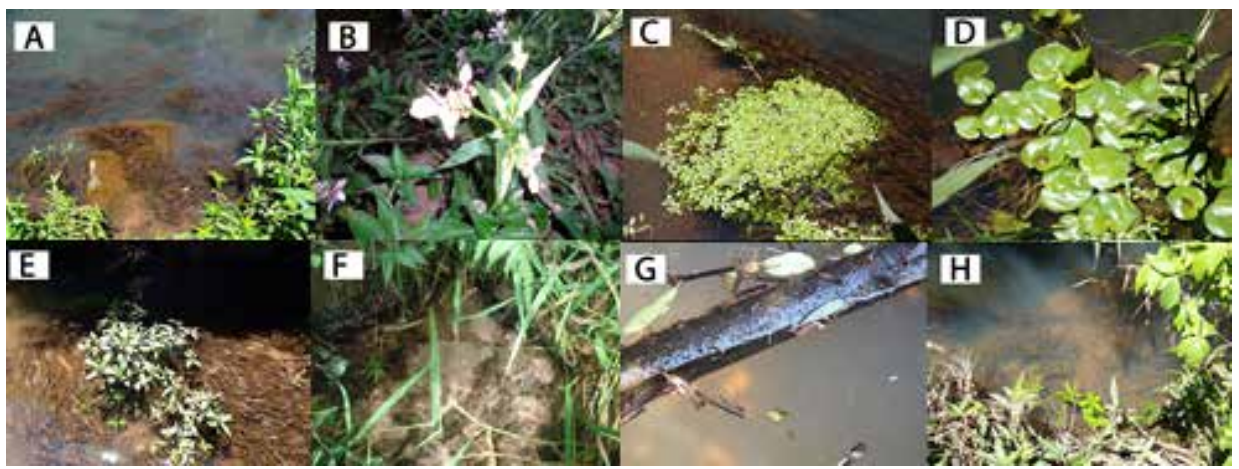

Figura 2. Macrófitas aquáticas do Arroio do Pinheiro Torto. A = Egeria dens $a ; \mathrm{B}=$ Tripogandra diuretica $; \mathrm{C}=$ Micranthemum micranthemoides $; \mathrm{D}=$ Heteranthera reniformis $; \mathrm{E}=$ Não identificada (Família Lamiaceae); F = Brachiaria plantaginea; $\mathrm{G}=$ Lemna minor; $\mathrm{H}=$ Ipomoea cairica Fonte: Dados da pesquisa

Quanto aos dados de frequência, a espécie Tripogandra diuretica mostrou-se a mais frequente, sendo encontrados espécimes desde a coluna d'água, estendendo-se pela encosta ao longo de toda a margem do trecho analisado, obtendo $100 \%$ de ocorrência na amostragem. Dentro do leito do arroio, foi mais frequente a espécie Egeria densa, que apareceu 85 vezes $(\mathrm{N}=85 ; 42,5 \%)$, seguida da espécie Micranthemum micranthemoides $(\mathrm{N}=42 ; 21 \%)$. A espécie Heteranthera reniformis apareceu oito vezes $(\mathrm{N}=8 ; 4 \%)$, enquanto a espécie Brachiaria plantaginea foi encontrada quatro vezes $(\mathrm{N}=4 ; 2 \%)$. Já as espécies de Lamiaceae não identificada, Lemna minor e Ipomoea cairica apareceram uma vez cada $(\mathrm{N}=1 ; 0,5 \%)$.

A análise básica de substrato apresentou um $\mathrm{pH}$ baixo $(5,4)$; bem como baixa quantidade de argila (10,4\%), de matéria orgânica (1,4\%) e baixa porcentagem de bases (69\%). A Capacidade de Troca de Cátions (CTC) está entre os valores considerados médios $\left(7,1 \mathrm{cmol}_{\mathrm{c}} / \mathrm{dm}^{3}\right)$. Os teores de $\mathrm{P}\left(107,9 \mathrm{mg} / \mathrm{dm}^{3}\right)$ foram classificados como muito altos para solos alagados e $\mathrm{K}\left(170 \mathrm{mg} / \mathrm{dm}^{3}\right)$ como alto. $\mathrm{A}$ análise de micronutrientes revelou que $\mathrm{Mn}, \mathrm{Zn}$ e $\mathrm{Cu}$ estão acima dos valores de referência (Quadro 1). 
Quadro 1. Análise de solo de macronutrientes e micronutrientes no Arroio do Pinheiro Torto na área da RPPN Maragato

\begin{tabular}{|c|c|c|c|c|c|c|c|c|c|}
\hline \multicolumn{5}{|c|}{ MACRONUTRIENTES } & \multicolumn{5}{c|}{ MICRONUTRIENTES } \\
\hline $\mathrm{P}$ & $\mathrm{K}$ & $\mathrm{S}$ & $\mathrm{Al}$ & $\mathrm{Ca}$ & $\mathrm{Mg}$ & $\mathrm{B}$ & $\mathrm{Mn}$ & $\mathrm{Zn}$ & $\mathrm{Cu}$ \\
\hline \multicolumn{3}{|c|}{$\mathrm{mg} / \mathrm{dm}^{3}$} & \multicolumn{3}{c|}{$\mathrm{cmol} / \mathrm{cm}^{3}$} & \multicolumn{5}{c|}{$\mathrm{mg} / \mathrm{dm}^{3}$} \\
\hline 107,9 & 170 & $>50,0$ & 0,0 & 2,7 & 1,7 & 0,2 & 96,6 & 9.60 & 7,12 \\
\hline
\end{tabular}

Fonte: Dados da pesquisa

A análise de macronutrientes e micronutrientes, em tecido vegetal da espécie mais frequente no leito, foi realizada com a espécie Egeria densa, revelando (em comparação às espécies cultiváveis e forrageiras), altos valores de $\mathrm{N}, \mathrm{Fe}, \mathrm{Mn} \mathrm{e}$ Zn (Quadro 2).

Quadro 2. Análise de tecido vegetal da espécie Egeria densa, no Arroio do Pinheiro Torto na área da RPPN Maragato

\begin{tabular}{|c|c|c|c|c|c|c|c|c|c|c|}
\hline \multicolumn{7}{|c|}{ MACRONUTRIENTES } & \multicolumn{5}{c|}{ MICRONUTRIENTES } \\
\hline $\mathrm{N}$ & $\mathrm{P}$ & $\mathrm{K}$ & $\mathrm{Ca}$ & $\mathrm{Mg}$ & $\mathrm{S}$ & $\mathrm{Zn}$ & $\mathrm{Cu}$ & $\mathrm{Mn}$ & $\mathrm{Fe}$ & $\mathrm{B}$ \\
\hline \multicolumn{9}{|c|}{$\%$} & \multicolumn{6}{c|}{$\mathrm{mg} / \mathrm{kg}$} \\
\hline 3,24 & 0,48 & 0,94 & 0,44 & 0,05 & 0,17 & 147,51 & 26,15 & $1.958,1$ & $2.394,2$ & 7,12 \\
\hline
\end{tabular}

Fonte: Dados da pesquisa

\section{DISCUSSÕES}

Os bioindicadores fornecem informações sobre os efeitos ecológicos de poluentes (RINGELBERG, 1983). O desenvolvimento de macrófitas aquáticas é influenciado principalmente por fatores físicos como geomorfologia, sedimento, clima e hidrologia (THOMAZ, 2002); fatores abióticos como temperatura, vento, precipitação, disponibilidade de luz, características do substrato, concentração de nitrogênio e fósforo, condutividade, $\mathrm{pH}$ e variação do nível da água; além de interações bióticas tais como competição, herbivoria e potencial de dispersão e colonização de cada espécie (LACOUL; FREEDMAN, 2006). Entretanto, os fatores 
que mais contribuem para o crescimento e a proliferação das macrófitas aquáticas são os de origem antrópica como o excesso de nutrientes provenientes de esgoto doméstico, a erosão de terras agrícolas e os resíduos industriais (MITCHELL, 1974).

Neste estudo em ambiente lótico foram encontradas oito espécies de macrófitas aquáticas de oito famílias, uma diversidade baixa quando comparada a estudos em ambientes lênticos no Rio Grande do Sul, sendo um dos motivos a velocidade da correnteza. Na área urbana do município de Guaíba, às margens do lago Guaíba, Lisboa e Gastal (2003) obtiveram como resultado 37 espécies de 24 famílias. O estudo de Mauhs et al. (2006), em uma área de banhado na planície costeira do Rio Grande do Sul, resultou em 18 espécies de macrófitas aquáticas, destacando-se em riqueza as famílias Cyperaceae (seis) e Poaceae (três), sendo as demais famílias representadas por uma única espécie. Trindade et al. (2010), no levantamento da vegetação aquática do campus Carreiros da Universidade Federal do Rio Grande (FURG), revelaram a presença de 43 espécies de macrófitas aquáticas distribuídas em 21 famílias botânicas.

As espécies de macrófitas aquáticas Egeria densa, Lemna minor, Micranthemum micranthemoides, Ipomoea cairica, Tripogandra diuretica, Brachiaria plantaginea e Heteranthera reniformis, encontradas neste estudo, são consideradas daninhas, ou seja, plantas que infestam espontaneamente áreas com ocupação ou interferência humana (VARGAS; ROMAN, 2008), também chamadas de plantas invasoras (LORENZI, 1991). Segundo este último autor, suas sementes podem mover-se pela água de enxurradas, chegando a riachos naturais, rios e canais de irrigação e drenagem, onde, em alguns casos, tendem a quebrar a dormência e aumentar a germinação. Assim, instalam-se e desenvolvem-se nestes locais quando encontram condições propícias na água e no solo.

Das espécies encontradas duas são classificadas como submersas, duas como flutuantes e quatro como anfíbias. Cheruvelil e Sorrano (2008), Lacoul e Freedman (2006) e Pozo et al. (2011) observaram em seus estudos que a ocorrência de macrófitas emergentes e flutuantes em ambientes aquáticos pode estar relacionada ao uso e ocupação do solo com atividades antropogênicas, especialmente com a agricultura, já que estas contribuem consideravelmente com o acúmulo de macronutrientes e micronutrientes na água. Nutrientes, em especial N e P, também têm sido apontados 
como importantes determinantes da diversidade e composição de comunidades de macrófitas aquáticas (ESTEVES, 2011).

A natureza do fundo dos cursos d'agua é também um importante determinante das plantas que podem estabelecer-se ali, pois há fatores que implicam na sua fixação e sobrevivência como, por exemplo, quantidade de matéria orgânica que se deposita (BENNET; HUMPHRIES, 1974).

A espécie mais frequente, Tripogandra diuretica, tem a capacidade de infestar principalmente pastagens, jardins, lavouras perenes e margens de canais, apresentando nítida preferência por locais úmidos e sombreados (LORENZI, 1991), o que justifica sua alta presença desde a margem do arroio a uma larga faixa da encosta.

Egeria densa, a espécie mais frequente no leito do arroio, tem a tendência de dominar o ambiente devido ao seu crescimento vigoroso, produzindo grandes infestações sem permitir o desenvolvimento de outras espécies (COOK; URMIKÖNIG, 1984), o que explica sua grande presença na área de estudo em questão. O aumento da eutrofização da água propicia grande desenvolvimento vegetativo dessa planta (LORENZI, 1991), o que é um forte indício de alteração na qualidade das águas do Arroio do Pinheiro Torto.

Segundo Rozema (1998), há metais essenciais que, quando em excesso, podem ser tóxicos, tais como cobalto, cobre, ferro, manganês, molibdênio, selênio e zinco. Os metais ocorrem em corpos d'água principalmente devido a despejos decorrentes de processos industriais, na forma de substâncias químicas orgânicas e/ ou inorgânicas (DERISIO, 2000).

A alta concentração de ferro nos tecidos vegetais de Egeria densa pode indicar que há alta quantidade deste nutriente também nas águas do Arroio do Pinheiro Torto. A coloração marrom-avermelhada dos espécimes encontrados em tal arroio também pode estar relacionada ao excesso de óxidos de ferro. A presença de ferro na água está relacionada aos processos de erosão das margens, a efluentes industriais, especialmente por indústrias metalúrgicas pela remoção da camada oxidada (ferrugem) das peças (CARIS et al., 2008). O padrão de potabilidade estabelecido pela Portaria $\mathrm{n}^{0}$ 2914/2011, do Ministério da Saúde, é de 0,3 mg/L, enquanto a análise em tecidos vegetais revelou valor muito elevado, 2394,2 mg/kg. 
O comportamento do Mn nas águas é muito semelhante ao do ferro, desenvolvendo coloração negra na água (CARIS et al., 2008). A concentração permitida pela mesma Portaria é menor que $0,1 \mathrm{mg} / \mathrm{L}$; e encontrou-se uma quantidade bem superior, de 1958,1 $\mathrm{mg} / \mathrm{kg}$ nos tecidos dos espécimes. Em águas naturais as altas concentraçóes de zinco se devem a despejos industriais (DERISIO, 2000). Para o zinco, $5,0 \mathrm{mg} / \mathrm{L}$ é o valor máximo permissível e na amostra de tecido de Egeria densa obteve-se o valor de $147,51 \mathrm{mg} / \mathrm{kg}$.

Altas concentrações de N e P na água indicam poluição (MALIK; NADEM, 2011). O aumento desses nutrientes na água é, em parte, do uso de fertilizantes na agricultura (VON SPERLING, 2011). Alguns estudos mostram que a concentração desses nutrientes no sedimento e na água tem proporcionado maior abundância e produtividade de macrófitas aquáticas, assim como maior ocorrência de espécies flutuantes em locais eutróficos (HENRY-SILVA et al., 2008; THOMAZ et al., 2006). As plantas assimilam o fosfato diretamente do solo ou da água e o incorporam diretamente em compostos orgânicos diversos (RICKLEFS, 2003).

Dois caminhos principais têm sido explorados em relação às macrófitas aquáticas como bioindicadoras: a capacidade de absorverem o excesso de nutrientes e poluentes da água na qual estão crescendo e a capacidade dos ecossistemas dominados por plantas aquáticas remover substâncias poluentes da água fluindo através deles (MITCHELL, 1974). Em um estudo realizado por Lopes-Ferreira (2000) no reservatório Salto Grande (SP), macrófitas reduziram 50\% de nitrito, $63 \%$ de fosfato inorgânico, $32 \%$ de fósforo e $90 \%$ da densidade de coliformes fecais da água deste. Alguns autores (FISHER; ACREMAN, 2004; CRONIN et al., 2006; VERHOVEN et al., 2006) destacam que as macrófitas aquáticas estocam quantidades de nutrientes em sua biomassa, o que foi verificado com a análise de tecido vegetal de Egeria densa neste estudo.

Segundo Pedralli (2003), o biomonitoramento por meio de plantas aquáticas pode ser realizado pelo indicativo de sua presença ou ausência, como também pela análise de parâmetros, tais como tamanho da população ou comunidade, forma e atributos funcionais quantificáveis, geralmente morfológicos, de fácil visualização e medição no campo. Ainda recomenda o emprego das formas biológicas anfíbias, emergentes, submersas fixas e flutuantes fixas, desde que apresentem características 
como serem sedentárias, acumuladoras de poluentes, com longo ciclo de vida, abundantes, conhecidas taxonomicamente, resistentes à manipulação e transporte, de fácil manutenção e coleta.

A presença de macrófitas aquáticas em ambientes lóticos, assim como em ambientes lênticos, está relacionada a diversos fatores e sua presença é de extrema importância nestes ecossistemas devido às suas diversas funções e, especialmente, ao seu potencial de absorverem o excesso de nutrientes e poluentes das águas. Análises de solo e de tecidos vegetais são instrumentos que auxiliam na determinação da composição e das propriedades dos recursos hídricos, revelando a deficiência ou excesso de macronutrientes e micronutrientes, que em ambos os casos são prejudiciais aos seres vivos.

Conclui-se que devido à ausência de macrófitas aquáticas no Arroio do Valinho, as águas deste recurso hídrico não apresentaram quantidades significativas de macronutrientes ou micronutrientes, o que sugere uma qualidade aparentemente boa, podendo estar relacionado ao uso do solo (ambiente florestal preservado). Já o Arroio do Pinheiro Torto, o qual recebe resíduos domésticos e industriais do município e tem interferência de atividades agrícolas no entorno, pode estar com a qualidade de suas águas alterada e com excesso de alguns macronutrientes e micronutrientes (comprovado pela análise de solo e tecido vegetal), o que vem permitindo o desenvolvimento e a proliferação de macrófitas aquáticas, algumas em grande quantidade.

Portanto, esta é uma análise complementar aos parâmetros físicos, químicos e biológicos de qualidade de água, os quais não podem ser descartados nem substituídos para se estabelecer de fato uma conclusão sobre padrões de qualidade. Recomenda-se, assim, maiores estudos destas águas, inclusive deste estudo, levandose em consideração a sazonalidade, que pode interferir nos resultados de riqueza de espécies e, ainda, ao poder público a adoção de medidas quanto à eliminação de esgoto doméstico e industrial nos arroios do município e fiscalização em relação às indústrias, para fins de conservação dos recursos hídricos e melhoria da qualidade das águas. 


\section{AGRADECIMENTOS}

Ao Sr. Rogério Benvegnú Guedes, Proprietário e Gestor da RPPN Maragato pela autorização da pesquisa na RPPN. À Associação do Sindicato dos Trabalhadores nas Indústrias da Construção e do Mobiliário de Passo Fundo pela autorização de entrada na área. Aos Srs. Rogério Benvegnú Guedes e Antônio Rogério Spindola pelo auxílio como guias de trilha. Às Esp. Angela Bedin, Andréa Aline Mombach e Gabriela Cássia Consalter pela ajuda nos trabalhos de campo, críticas e sugestões. À Ms. Branca Maria Aimi Severo e equipe do Herbário da Universidade de Passo Fundo; à Ms. Gisele Sana Rebelatto e ao Dr. Jean Bucke com a identificação das espécies. Ao Eng. Agrônomo Volnei de Moura Fão e equipe do Laboratório de Solos, bem como à Universidade de Passo Fundo, pelas análises de substrato e tecido vegetal. Ao Eng. Agrônomo Ademir Trombetta pelo auxílio às análises. Ao PhD Miguel Ângelo Marini pelas sugestões e críticas de uma versão anterior do manuscrito.

\section{REFERÊNCIAS}

AGENCES DE L'EAU. Étude bibliographique des méthodes biologiques d'évaluation de la qualité des eaux de surface continentales. Synthèse bibliographique. Etude Inter Agences no 35. I.D.E. Environnement, 1993.

BARBOUR, M. T.; GERRITSEN, J.; SNYDER, B. D.; ST RIBLING, J. B. Rapid bioassessment protocols for use in streams and wadeable rivers: periphyton, benthic macroinvertebrates and fish. 2. ed. Washington, D.C.: Environmental Protection Agency; Office of Water; 1999. (EPA 841-B-99-002).

BENNETT, D. P.; HUMPHRIES, D. A. Introduccion a la ecologia de campo. Madrid: Blume, 1974.

BRASIL. Ministério da Saúde. Portaria n ${ }^{0} 2.914$, de 12 de dezembro de 2011. Dispõe sobre os procedimentos de controle e de vigilância da qualidade da água para consumo humano e seu padrão de potabilidade. Diário Oficial da União, n. 3, de 04 de janeiro de 2012, Seção 1, página 43. 
BRASIL. Instituto Chico Mendes de Conservação da Biodiversidade. Portaria $\mathbf{n}^{\mathbf{o}}$ 14, de 15 de outubro de 2007. Aprova criação da RPPN - Reserva Particular do Patrimônio Natural Maragato. DOU, 198 Brasília: 15 out. 2007, Seção I, p. 75-76.

BRASIL. Instituto Chico Mendes. Plano de Manejo da Reserva Particular do Patrimônio Natural - RPPN Maragato. Brasília: Instituto Chico Mendes, 2011.

CALLISTO, M.; GONÇALVES, J. F. Jr. A vida nas águas das montanhas. Ciência Hoje, v. 31, n. 182, p. 68-71, 2002.

CALLISTO, M.; GONÇALVES Jr., J. F.; MORENO, P. Invertebrados aquáticos como bioindicadores. In: GOULART, E. M. A. (Org.) Navegando o Rio das Velhas das Minas aos Gerais: Proj. Manuelzão. Belo Horizonte: Coopmed, 2005. p. 555-567.

CAMARGO, A. F. M.; FLORENTINO, E. R. Population dynamics and net primary production of the aquatic macrophyte Nymphaea rudgeana C. F. mey in a lotic environment of the Itanhaém River basin (SP, Brazil). Revista Brasileira de Biologia, São Carlos, v. 60, n. 1, p. 83-92, fev. 2000.

CARIS, M. E.; ANDRADE, A. S. P.; PHILIPPI, L. S. Determinação do potencial de biorremediação de nutrientes e bioindicação de águas residuárias da suinocultura por macrófitas flutuantes (Lemna minuta) - Efeito de altas taxas de nitrogênio amoniacal. PESQUISA Evidência, Joaçaba, v. 8, n. 1-2, p. 85-102, jan./dez. 2008.

CERVI, A. C.; HATSCHBACH, G.; GUIMARÃES, O. A. Nota prévia sobre plantas aquáticas (fanerogâmicas) do Estado do Paraná (Brasil). Bol. Mus. Bot. Mun., v. 58, p. $1-17,1983$.

CERVI, A. C.; BONA, C.; MOÇO, M. C. C.; LINSINGEN, L. V. Macrófitas aquáticas do Município de General Carneiro, Paraná, Brasil. Biota Neotropica, v. 9, n. 3, p. 215222, 2009.

CHERUVELIL, K.; SORANNO, P. Relationships between lake macrophyte cover and lake an landscape features. Aquatic Botany, v. 88, p. 219-227, 2008.

COMISSÃO DE QUÍMICA E FERTILIDADE DO SOLO - RS/SC. Manual de adubação e calagem para os Estados do Rio Grande do Sul e Santa Catarina. 10. ed. Porto 
Alegre: [s.n.], 2004.

COOK, C. D. K.; URMI-KONIG, K. A revision of the genus Egeria (Hydrocharitaceae). Aquatic Botany, v. 19, p. 73-96, 1984.

CORDAZZO, C. V.; SEELIGER, U. Guia ilustrado da vegetação costeira no extremo sul do Brasil. Rio Grande: FURG, 1988.

CORTES, R.; PINTO, P.; FERREIRA, M. T.; MOREIRA, I. Qualidade biológica dos ecossistemas fluviais. In: MOREIRA, I.; FERREIRA, M. T.; CORTES, R. M. V.; PINTO, P.; ALMEIDA, P. R. (Ed.). Ecossistemas Aquáticos e Ribeirinhos. Instituto da Água, Ministério das Cidades, Ordenamento do Território e Ambiente, Lisboa, 2002.

CRONIN, G.; LEWIS, W. M.; SCHIEHESER, M. A. Influence of freshwater macrophytes on the litoral ecosystem structure and function of young Colorado Reservoir. Aquactic Botany, v. 85, p. 37-46, 2006.

DERISIO, J. C. Introdução ao controle de poluição ambiental. 2. ed. São Paulo: Signus, 164 p. 2000.

DINIZ, C. R.; CEBALLOS, B. S. O.; BARBOSA, J. E. L.; KONIG, A. Uso de macrófitas aquáticas como solução ecológica para melhoria da qualidade de água. R. Bras. Eng. Agríc. Ambiental, Campina Grande, Suplemento, p. 226-230, 2005.

ESTEVES, F. A. Fundamentos de limnologia. Rio de Janeiro: Interciência; FINEP, 1998.

ESTEVES, F. A. (Coord.). Fundamentos de limnologia. 3. ed. Rio de Janeiro: Interciência, 2011.

ESTEVES, F. A.; CAMARGO, A. F. M. Sobre o papel das macrófitas aquáticas na estocagem e ciclagem de nutrientes. Acta Limnol. Bral., v. 1, p. 273-298, 1986.

FIGUEROA, F. E. V. Avaliação econômica de ambientes naturais: o caso das áreas alagadas: uma proposta para a represa do Lobo (Broa). 1996. 143f. Dissertação (Mestrado) - Universidade Federal de São Carlos, São Carlos, SP. 
FISHER, J.; ACREMAN, M. C. Wetland nutrient removal: a review of evidence. Hydrology and Earth System Sciences, v. 8, n. 4, p. 673-685, 2004.

FONTOURA, A. P. Elaboração de uma carta de qualidade biológica da água. Porto, Portugal: Universidade do Porto, 1984.

HENRY-SILVA, G. G.; CAMARGO, A. F. M.; PEZZATO, M. M. Growth of free-floating aquatic macrophytes in different concentrations of nutrients. Hydrobiologia: The Hague, v. 610, p. 153-160, 2008.

IRGANG, B. E.; GASTAL Jr., C. V. S. Plantas aquáticas da planície costeira do Rio Grande do Sul. Porto Alegre: Ed. dos Autores, 1996.

KOVÁCS, M. (Ed.). Biological Indicators in Environmental Protection. Tradução de Á. Máthé, Z. Tuba, J. L. Meenks, Zs. Csintalan. Chichester: Ellis Horwood, 1992. $125 \mathrm{p}$.

LACOUL, P.; FREEDMAN, B. Environmental influences on aquatic plants in freshwater ecosystems. Environmental Review, Denver, v. 14, n. 2, p. 89-136, 2006.

LÉVÊQUE, C. Ecosystèmes Aquatiques. Les Fondamentaux. Paris: Hachette Livre, 1996.

LISBOA, F. F.; GASTAL, C. V. S. Jr. Levantamento das macrófitas aquáticas na beira do Lago Guaíba no município de Guaíba, RS/Brasil. Caderno de Pesquisa Sér. Bio., Santa Cruz do Sul, v. 15, n. 1, p. 17-27, jan./jun. 2003.

LOPES-FERREIRA, C. M. Estudo de uma área alagada do rio Atibaia visando à elaboração de proposta de manejo para melhoria da qualidade da água no reservatório de Salto Grande (Americana, SP). 2000. 145 f. Tese (Doutorado) Universidade de São Paulo, São Paulo.

LORENZI, H. Plantas daninhas do Brasil: terrestres, aquáticas, parasitas e tóxicas. 2. ed. Nova Odessa: Instituto Plantarum de Estudos da Flora, 1991. 440 p.

LUCIANO, S. C. Macrófitas aquáticas Eichbornia azurea (Kunth) e Brachiaria 
arrecta (Stent). 1996. 155 f. Dissertação (Mestrado) - Universidade de São Paulo, Escola de Engenharia de São Carlos, SP.

MAGALHÃES, A. P. Jr. Indicadores ambientais e recursos hídricos: realidade e perspectivas para o Brasil a partir da experiência francesa. Rio de Janeiro: Bertrand Brasil, 2007. 686p.

MALIK, R. N.; NADEM, M. Spatial and temporal characterization of trace elements and nutrients in the Rawal Lake Reservoir, Pakistan using multivariate analysis techniques. Environ Geochem and Health, Dordrecht, v. 33, p. 525-541, 2011.

MANFRINATO, E. S. Avaliação do método Edafo-Fitodepuração para tratamento preliminar de águas. 1989. 98 f. Dissertação (Mestrado) - Universidade de São Paulo, Escola Superior de Agricultura Luiz de Queiroz ESALQ, Piracicaba, SP.

MAUHS, J.; MARCHIORETTO, M. S.; BUDKE, J. C. Riqueza e biomassa de macrófitas aquáticas em uma área úmida na planície costeira do Rio Grande do Sul, Brasil. São Leopoldo, Instituto Anchietano de Pesquisas, Botânica, n. 57, p. 289-302, 2006.

MITCHELL, D. S. Aquatic Vegetation and its use and control. Unesco, Paris, 1974. $135 \mathrm{p}$.

MORAES, A. R. Estimativa do estoque de elementos químicos em macrófitas aquáticas do reservatório de Salto Grande (Americana-SP). 1999. $94 \mathrm{f}$. Dissertação (Mestrado) - Universidade de São Paulo, Escola de Engenharia de São Carlos, São Carlos.

NOTARE, M. Plantas hidrófilas e seu cultivo em aquários. Rio de Janeiro: Sulamérica, 1992.

PEDRALLI, G. Macrófitos aquáticos: técnicas e métodos de estudos. Estudos de Biologia, v. 26, p. 5-24, 1990.

PEDRALLI, G. Macrófitas aquáticas como bioindicadoras da qualidade da água: alternativas para usos múltiplos de reservatórios. In: THOMAZ, S. M.; BINI, L. M. 
Ecologia e manejo de macrófitas aquáticas. Maringá: EDUEM, 2003. p. 171-188.

POMPÊO, M. L. M.; MOSCHINI-CARLOS, V. Macrófitas aquáticas e perifíton: aspectos ecológicos e metodológicos. São Carlos: Rima, 2003.

POTT, V. J.; POTT, A. Plantas aquáticas do Pantanal. Brasília: Embrapa, 2000.

POZO, R.; FERNÁNDEZ-ALÁEZ, C.; FERNÁNDEZ-ALÁEZ, M. The relative importance of natural and anthropogenic effects on community composition of aquatic macrophytes in Mediterranean ponds. Marine and Freshwater Research, v. 62, n. $2,2011$.

RICKLEFS, R. E. A economia da natureza. 5. ed. Rio de Janeiro: Guanabara Koogan, 2003. 503p.

RINGELBERG, J. General Remarks with Regard to Biological Indicators Used in Water Pollution Studies. In: BEST, E. P. H.; HAECK, J. (Ed.). Ecological indicators for the of air, water, soil, and ecosystems. Dordrecht: D. Reidel Publishing Company, 1983. p. 317-319.

ROZEMA, T. Toxicidade de metais pesados em humanos. Revista de Oxidologia, p. 22-30, nov./dez. 1998.

SCREMIN-DIAS, E.; POTT, V. J.; HORA, R. C.; SOUZA, P. R. Nos jardins submersos da Bodoquena: guia para identificação de plantas aquáticas de Bonito e região. Campo Grande: Ed. da UFMS. 1999. 160 p.

TEDESCO, M. J.; GIANELLO, C.; BISSANI, C. A.; BOHNEN, H.; VOLKWEISS, S. J. Análise de solo, plantas e outros materiais. 2. ed. Porto Alegre: Universidade Federal do Rio Grande do Sul, 1995. 174 p.

THOMAZ, S. M. Fatores ecológicos associados à colonização e ao desenvolvimento de macrófitas aquáticas e desafios de manejo. Planta daninha, Rio de Janeiro, v. 20, Edição Especial, p. 21-33, 2002.

THOMAZ, S. M.; BINI, L. M. Ecologia e manejo de macrófitas aquáticas. Maringá: 
Universidade Estadual de Maringá, 2003.

THOMAZ, S. M.; PAGIORO, T. A.; BINI, L. M.; MURPHY, K. J. Effect of reservoir drawdown on biomass of three species of aquatic macrophytes in a large sub-tropical reservoir (Itaipu, Brazil). Hydrobiologia: The Hague, v. 570, p. 53-59, 2006.

TRINDADE, C. R. T.; PEREIRA, S. A.; ALBERTONI, E. F.; PALMA-SILVA, C. Caracterização e importância das macrófitas aquáticas com ênfase nos ambientes límnicos do Campus Carreiros - FURG, Rio Grande, RS. Cadernos de Ecologia Aquática, v. 5, n. 2, p. 1-22, 2010.

VARGAS, L.; ROMAN, E. S. (Coord.). Manual de manejo e controle de plantas daninhas. Passo Fundo: Embrapa Trigo, 2008. 779p.

VERHOEVEN, J. T. A.; ARHEIMER, B.; YIN, C.; HEFTING, M. M. Regional and global concerns over wetlands and water quality. Trends in Ecology and Evolution, v. 21, n. 2, p. 96-103, 2006.

VON SPERLING, M. Introdução à qualidade das águas e ao tratamento de esgotos. Belo Horizonte: DESA/UFMG, 2011.

WETZEL, R. G. Limnologia. 2. ed. Lisboa: Fundação Calouste Gulbenkian, 1993. $1129 \mathrm{p}$.

Recebido em: 29 de setembro de 2014 Aceito em: 23 de setembro de 2015 